\title{
Tobramycin and bicarbonate synergise to kill planktonic Pseudomonas aeruginosa, but antagonise to promote biofilm survival
}

\author{
Karishma S Kaushik ${ }^{1,2}$, Jake Stolhandske ${ }^{3}$, Orrin Shindell ${ }^{3}$, Hugh D Smyth ${ }^{4}$ and Vernita D Gordon ${ }^{2,3}$
}

Increasing antibiotic resistance and the declining rate at which new antibiotics come into use create a need to increase the efficacy of existing antibiotics. The aminoglycoside tobramycin is standard-of-care for many types of Pseudomonas aeruginosa infections, including those in the lungs of cystic fibrosis (CF) patients. P. aeruginosa is a nosocomial and opportunistic pathogen that, in planktonic form, causes acute infections and, in biofilm form, causes chronic infections. Inhaled bicarbonate has recently been proposed as a therapy to improve antimicrobial properties of the CF airway surface liquid and viscosity of CF mucus. Here we measure the effect of combining tobramycin and bicarbonate against $P$. aeruginosa, both lab strains and CF clinical isolates. Bicarbonate synergises with tobramycin to enhance killing of planktonic bacteria. In contrast, bicarbonate antagonises with tobramycin to promote better biofilm growth. This suggests caution when evaluating bicarbonate as a therapy for CF lungs infected with $P$. aeruginosa biofilms. We analyse tobramycin and bicarbonate interactions using an interpolated surface methodology to measure the dose-response function. These surfaces allow more accurate estimation of combinations yielding synergy and antagonism than do standard isobolograms. By incorporating predictions based on Loewe additivity theory, we can consolidate information on a wide range of combinations that produce a complex dose-response surface, into a single number that measures the net effect. This tool thus allows rapid initial estimation of the potential benefit or harm of a therapeutic combination. Software code is freely made available as a resource for the community.

npj Biofilms and Microbiomes (2016) 2, 16006; doi:10.1038/npjbiofilms.2016.6; published online 25 May 2016

\section{INTRODUCTION}

A worldwide increase in antibiotic resistance has prompted the search for newer therapeutic strategies, including adjunct treatment approaches that can extend the lifetime of current antibiotics. ${ }^{1}$ Pseudomonas aeruginosa is an opportunistic and nosocomial human pathogen and multi-drug resistant strains are on the rise. ${ }^{2} P$. aeruginosa causes both acute and chronic infections. ${ }^{3}$ Acute $P$. aeruginosa infections are found in burn wounds and surgical sites, and are often initiated by planktonic bacteria. Acute $P$. aeruginosa infections are often resistant to antibiotic treatment and can result in delayed healing, sepsis and death. ${ }^{4}$ For cases where infection transitions from acute to chronic states, planktonic bacteria typically organise to develop biofilm structures. ${ }^{5}$ Chronic $P$. aeruginosa infections are found in chronic wounds in patients with diabetes ${ }^{6}$ and in the lungs of patients with cystic fibrosis $(\mathrm{CF})^{7,8}$ and chronic obstructive pulmonary disease, and are caused by multicellular biofilm aggregates. In CF patients, early $P$. aeruginosa infections are planktonic, intermittent and susceptible to antimicrobial therapy. During long-term infection, it has been suggested both that infecting $P$. aeruginosa converts to the biofilm phenotype and that infecting $P$. aeruginosa persists as a slow-growing, airway-adapted, stationary-phase population; either of these scenarios results in a chronic infection that is notoriously recalcitrant to antibiotic therapy. ${ }^{9-11}$
For both planktonic and biofilm $P$. aeruginosa infections, aminoglycosides are a well-established standard of care. ${ }^{12}$ For $\mathrm{CF}$, the inhaled aminoglycoside tobramycin is widely used as a long-term therapy. Tobramycin has toxic side effects on auditory and kidney function. ${ }^{13}$ Ototoxic side effects include dizziness, tinnitus and irreversible hearing loss. ${ }^{13}$ Nephrotoxic side effects, though reversible, can lead to renal insufficiency, and are exacerbated with prolonged duration of therapy. ${ }^{13}$ Reducing the dose and duration of tobramycin needed for clinical benefit would reduce toxic side effects. It has recently been shown that alkaline $\mathrm{pH}$, mediated by biogenic bases produced by bacteria or exogenous alkalis such as bicarbonate, may enhance the efficacy of aminoglycosides. ${ }^{14-16}$

Independently, the base bicarbonate has an important place in the pathology of CF. In CF patients, a defect in the cystic fibrosis transmembrane conductance regulator (CFTR) impairs bicarbonate transport, leading to acidification of the lung. ${ }^{17-19}$ In a newborn CF pig model, ${ }^{20}$ low pH of the airway surface liquid reduces the activity of innate antimicrobial factors, but antimicrobial efficacy could be restored by aerosolising bicarbonate into the lung. In addition, CF lungs have thick, sticky mucus and impaired mucociliary transport; these promote the growth of bacterial infections. ${ }^{21}$ It was recently proposed that bicarbonate, by chelation of cationic bridges, could help thin mucus, for better

${ }^{1}$ Department of Molecular Biosciences, University of Texas, Austin, TX, USA; ${ }^{2}$ Institute of Cellular and Molecular Biology, University of Texas, Austin, TX, USA; ${ }^{3}$ Center for Nonlinear Dynamics and Department of Physics, University of Texas, Austin, TX, USA and ${ }^{4}$ Division of Pharmaceutics, College of Pharmacy, University of Texas, Austin, TX, USA. Correspondence: VD Gordon (gordon@chaos.utexas.edu)

Received 16 November 2015; revised 12 April 2016; accepted 14 April 2016 

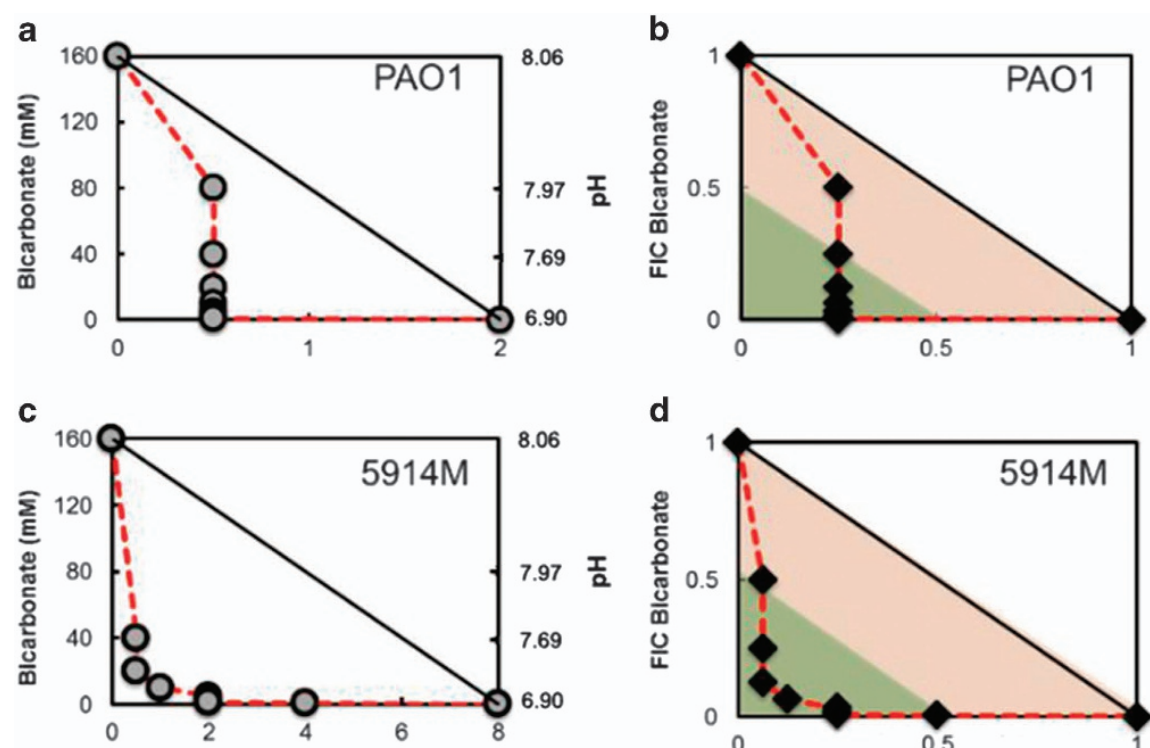

d
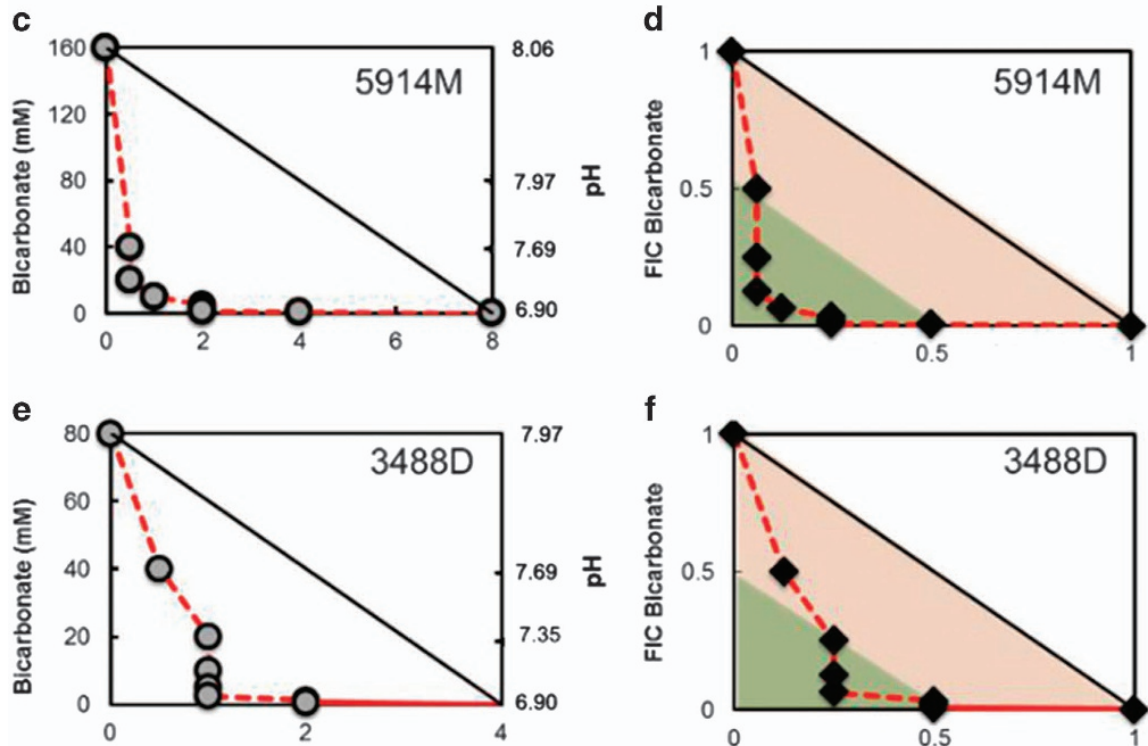

f
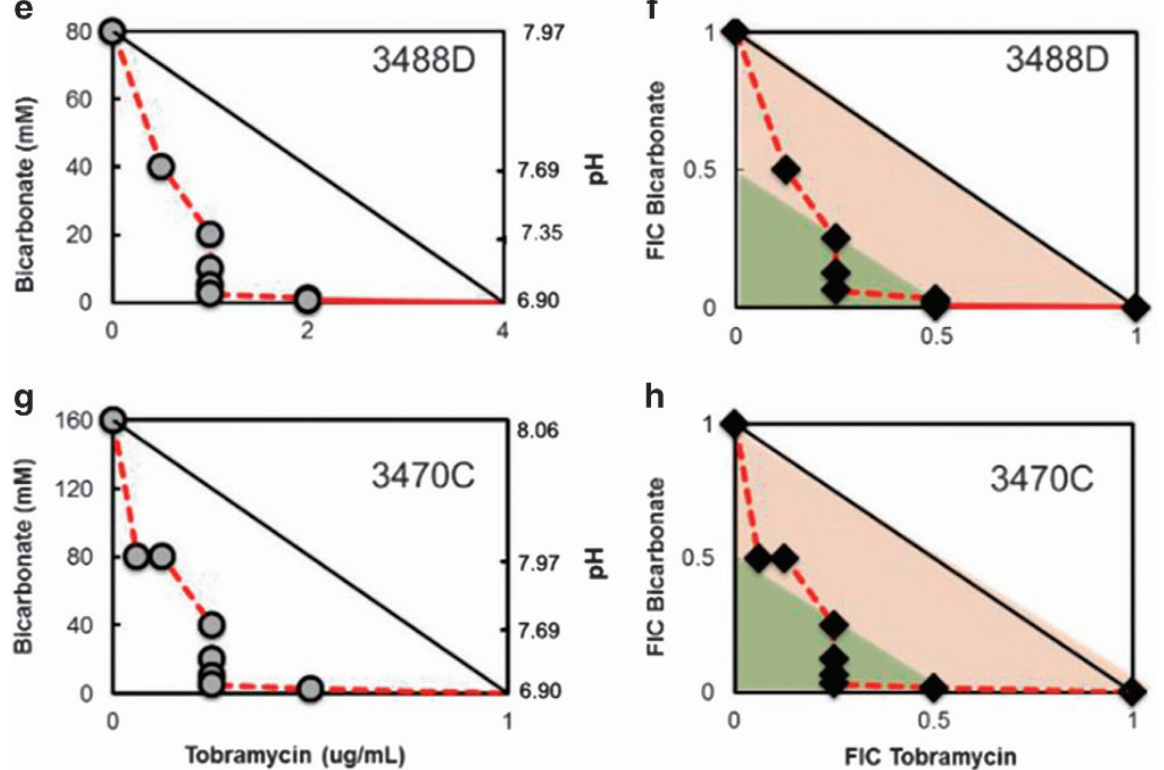

Figure 1. Isobologram analyses for tobramycin-bicarbonate combination against planktonic cells of $P$. aeruginosa (a, b) laboratory strain PAO1, and $P$. aeruginosa clinical cystic fibrosis isolates (c, d) 5914M (mucoid), (e, f) 3488D (dwarf) and (g, h) 3470C (classic). A strong synergisticadditive effect is observed against all four strains. Points along the isobologram represent the growth-no growth interface. (b, $\mathbf{d}, \mathbf{f}, \mathbf{h})$ The orange shaded area represents the additive region and the green shade area represents the synergistic region.

clearance. $^{22}$ Therefore, in clinical trials, inhaled bicarbonate is being evaluated as a potential therapeutic approach for CF patients. $^{22}$

Here we evaluate the potential of bicarbonate as an adjunctive therapy to enhance the efficacy of tobramycin against planktonic and biofilm $P$. aeruginosa. We find that the combination of bicarbonate and tobramycin shows a strong synergistic effect against planktonic $P$. aeruginosa, for both laboratory strains and clinical CF isolates. Synergy not only reduces the concentration of tobramycin required to kill $P$. aeruginosa cells but, for some strains, also enhances the rate of killing. However, for biofilms of $P$. aeruginosa, the combination of tobramycin and bicarbonate is markedly antagonistic-i.e., the biofilm survives treatment with the combination better than it survives treatment with the corresponding concentration of tobramycin or bicarbonate alone. Although the synergistic effect against planktonic $P$. aeruginosa cells holds promise, the antagonistic effect against biofilms prompts caution in the development of bicarbonate as a CF therapy.

We quantitatively analyse the synergy and antagonism observed using dose-response surfaces, ${ }^{23,24}$ which examine drug combinations in greater detail than do more traditional methods, and we make our software code for this available for use by the scientific community (Supplementary Material). This dose-response surface analysis allows a more in-depth study of the tobramycin+bicarbonate combinations to more accurately estimate regimes or synergy and antagonism. Furthermore, we can compare measured surfaces with surfaces predicted for additive interactions, ${ }^{25}$ to determine whether the net outcome of a wide range of combinations is additive, synergistic or antagonistic. For systems with complex dose-response curves that show synergy in some parameter spaces and antagonism in others, this kind of combined-surface analysis could be developed into a tool for rapid assessment and making treatment decisions. 


\section{RESULTS}

Combination of tobramycin and bicarbonate produces synergy against planktonic cells of $P$. aeruginosa

Using the checkerboard microdilution assays, we test combinations of tobramycin and bicarbonate against planktonic cells of a wide range of $P$. aeruginosa strains: lab strains PAO1 and PA14, four spontaneously generated antibiotic-resistant mutants in the PA14 background, and clinical isolates from CF patients. Cultures were grown overnight so that cells were in the stationary phase, and the next day these stationary-phase cells were added to different combinations of tobramycin and bicarbonate. We create isobolograms by plotting points describing the growth/no-growth interface such that the tobramycin concentration is the abscissa and the bicarbonate concentration is the ordinate. We calculated the $\Sigma F I C$ (fractional inhibitory concentration) index for each well along the growth/no-growth interface, where the lowest concentration of tobramycin alone or bicarbonate alone that results in no growth is defined as the minimum inhibitory concentration (MIC) for that substance. Addition of bicarbonate to tobramycin produces a synergistic-to-additive effect for all the strains tested (Figure 1 and Supplementary Figures S1-S6). Notably, even though a mild increase in $\mathrm{pH}$ from neutral conditions does not inhibit growth in the absence of tobramycin, these relatively small changes in $\mathrm{pH}$ in the presence of tobramycin produce a net synergistic effect. Supplementary Table S1 summarises the results for all strains tested. Supplementary Table S2 shows measured $\mathrm{pH}$ of LB medium at the relevant bicarbonate concentrations.

Further, for selected antibiotic-resistant and clinical strains, we evaluate combinations using a fixed concentration of bicarbonate. For this, the bicarbonate concentration associated with the lowest SFIC value for the select strain was plotted against varying tobramycin concentrations tested. For the strains thus examined, we find that the addition of bicarbonate reduces the concentration of tobramycin required to inhibit planktonic $P$. aeruginosa cells even at tobramycin concentrations much lower than that needed to produce synergy (Figure 2, Supplementary Figures S11-S13).

From these results, we infer that combination with bicarbonate may reduce the concentration of tobramycin needed for clinical benefit against acute infections associated with planktonic $P$. aeruginosa cells and may help treat tobramycin-resistant infections. If so, then this would reduce the dosage and ameliorate toxicity, associated with long-term tobramycin administration. For inhaled tobramycin powder, which is the standard of care for CF lung infections, a standard dose is $112 \mathrm{mg}$ twice daily. Reducing the amount of tobramycin needed for efficacy required would reduce the burden of inhaling so much powder.

Bicarbonate enhances the rate at which tobramycin kills planktonic $P$. aeruginosa cells

In addition to dosage, a reduction in the duration of tobramycin therapy would also help reduce toxic side effects and antibiotic selection pressure. Furthermore, understanding how the rate of killing is impacted by the addition of bicarbonate to tobramycin will be important for clinical translation. Standard in vitro studies are done without drug clearance; so that the concentration of drug is constant over time, and these will under-estimate the actual concentrations required in vivo, where drug clearance initiates as soon as the drug is administered. Thus, improving the rate of killing as well as the dose required for killing should give rise to additional benefits in vivo.

Therefore, we examine the rate at which the combination of tobramycin and bicarbonate produced inhibition of planktonic $P$. aeruginosa cells. To examine the time-kill kinetics of the tobramycin and bicarbonate combination, we use the lab strain PAO1, antibiotic-resistant strain 1 and the clinical isolate 5913C. Using the concentrations of tobramycin and bicarbonate that show the greatest synergy (i.e., the lowest $\Sigma F I C$ index) in the checkerboard microdilution assays, we measure the rate of killing of the combination and each agent alone (at combination concentration and $\mathrm{MIC})$ every $2 \mathrm{~h}$ for the first $8 \mathrm{~h}(0,2,4,6,8 \mathrm{~h}$ time points) and then at $24 \mathrm{~h}$.

For PAO1, treatment with a combination of $0.25 \mu \mathrm{g} / \mathrm{ml}$ tobramycin and $5 \mathrm{mmol}$ bicarbonate eradicates the population by $4 \mathrm{~h}$; in contrast, each agent alone does not kill the population in this time (Figure 3a). Notably, the timescale for killing by the combination is comparable to that for $2 \mu \mathrm{g} / \mathrm{ml}$ tobramycin, the MIC for this strain (Supplementary Figure S14A). This indicates that the combination reduces the concentration of tobramycin needed to eradicate the population by nearly $\times 10$.

For antibiotic-resistant strain \#1, treatment with a combination of $1 \mu \mathrm{g} / \mathrm{ml}$ tobramycin and $5 \mathrm{mmol}$ bicarbonate kills the population by $6 \mathrm{~h}$, while each agent alone does not kill the population in this time (Figure $3 \mathrm{~b}$ ). Treatment with $8 \mu \mathrm{g} / \mathrm{ml}$ tobramycin, this strain's MIC, takes $>6 \mathrm{~h}$ to kill the population (Supplementary Figure S14B). Thus, for this strain bicarbonate not only reduces the necessary concentration of tobramycin by $8 \times$, but also reduces the needed duration of exposure.

For the CF isolate strain 5913C, the synergistic combination of $1 \mu \mathrm{g} / \mathrm{ml}$ tobramycin and $5 \mathrm{mmol}$ bicarbonate begins killing only after $6 \mathrm{~h}$ of exposure; the population is entirely killed between 8 and $24 \mathrm{~h}$ after exposure to the combination (Figure $3 \mathrm{c}$ ). At $8 \mathrm{~h}$, the killing curve for the combination lags that of the tobramycin MIC by $7 \times$. (Supplementary Figure S14C). However, both treatments eradicate the population at the end of $24 \mathrm{~h}$, with the combination treatment affording a decrease in the concentration of tobramycin. Thus, for this strain the addition of bicarbonate reduces the concentration of tobramycin needed for killing, but at the cost of increasing the exposure time needed. These results reiterate that the effect of the combination of tobramycin and bicarbonate shows notable strain-to-strain variation.

For $P$. aeruginosa biofilms, the combination of tobramycin and bicarbonate is antagonistic

Although we observe notable strain-to-strain variation, combination of tobramycin and bicarbonate show a synergistic effect for planktonic cells of all strains tested. In the planktonic state, $P$. aeruginosa is typically associated with acute infections and sepsis, but chronic $P$. aeruginosa infections are typically biofilm in nature. Therefore, we evaluate the effects of the tobramycin and bicarbonate combination against in vitro, laboratory-grown biofilms using the lab strain PAO1 and CF clinical isolates 4219D and $3470 \mathrm{C}$. For all three strains, overnight, 24-h biofilms demonstrate high biomass and metabolic activity (Supplementary Figure S3). Results are summarised in Supplementary Table S3. In striking contrast to the case for their planktonic counterparts, these data show at best an additive effect, and at worst a strongly antagonistic effect. This is shown by FIC values $>1$ (Supplementary Table S3) and by isobolograms that extend to the right of the line of additivity (Figure 4). We speculate that the difference in the response of biofilms to combination treatment with tobramycin and bicarbonate, compared with the response of planktonic cells to combination treatment, may arise from acidification of the biofilm environment or limited diffusion into the interior of the biofilm. ${ }^{26,27}$ Alternatively, we may be detecting more metabolically active bacteria in the biofilm if the $\mathrm{pH}$ change induces otherwise-inactive bacteria into an active state.

Tobramycin+bicarbonate combinations have an additive effect against stationary-phase cells

To determine the degree to which the antagonism observed for the combination of tobramycin+bicarbonate against $P$. aeruginosa biofilms is a biofilm-specific phenomenon, distinct from an effect arising from the presence of high-density, stationary- 

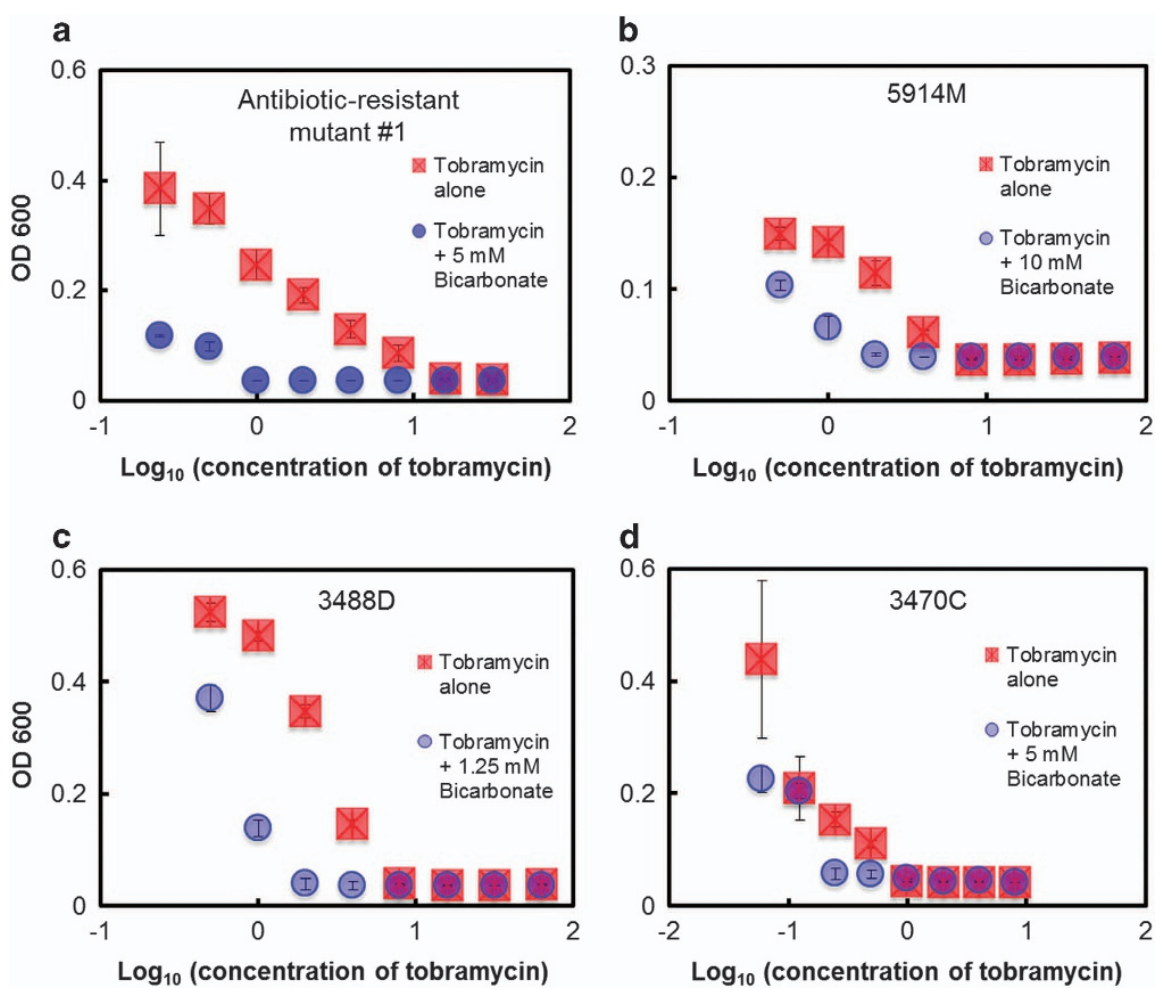

Figure 2. Synergy between fixed combinations of bicarbonate and tobramycin in killing $P$. aeruginosa (a) antibiotic-resistant mutant strain \# 1 and clinical CF isolates (b) 5914M, (c) 3488D and (d) 3470C. Error bars represent s.e.m.; $N=3$. For this, the bicarbonate concentration associated with the lowest $\Sigma F I C$ value for the select strain was plotted against varying tobramycin concentrations tested. For all four strains thus examined, addition of bicarbonate reduces the concentration of tobramycin required to inhibit planktonic $P$. aeruginosa cells even at tobramycin concentrations much lower than that needed to produce synergy.

phase cells, we performed the checkerboard assay with different combinations of tobramycin+bicarbonate using a high density of stationary-phase PAO1 cells, as described in more detail in the Materials and Methods section.

At this high density of cells, the efficacy of both tobramycin and bicarbonate was reduced. This is likely the result of the inoculum effect, or the per-cell concentration of antibiotic or antibacterial agent being more important for whether or not a cell is killed than is the absolute (Molar) concentration. As a result, we were unable to use a $90 \%$ threshold to describe MIC. Therefore, we used $\sim 50 \%$ inhibition of bacterial growth as the MIC for all subsequent analysis of planktonic, stationary-phase bacteria. Using isobologram analysis and FIC values (Supplementary Figure S15), we found an additive effect for tobramycin+bicarbonate combinations against stationary-phase PAO1 cells.

The antagonistic effect on $P$. aeruginosa biofilms is likely due to a combination of biofilm-specific properties and presence of stationary-phase cells

To examine the degree to which planktonic bacteria might be more susceptible to killing because of their exponential growth phase, as opposed to the quasi-stationary-phase state of many biofilm bacteria, we examined the lag phase of PAO1 bacteria introduced into different concentrations of tobramycin alone, bicarbonate alone and combinations of tobramycin and bicarbonate (Supplementary Figures S16-S18). In no case was the lag time shorter than 200 min (this was found for combinations that we had previously found to be well below the inhibitory threshold). Moreover, for combinations just below the growthno growth interface, bacteria did not re-enter exponential growth over the time of observation $(360 \mathrm{~min})$. This suggests that some killing may happen before early exponential phase begins-i.e., some killing happens at some point in the lag phase characterising the dilution from stationary phase into fresh medium.

We recall our finding that stationary-phase bacteria are less susceptible to tobramycin-bicarbonate combinations than are diluted (and subsequently exponentially growing) bacteria, but that stationary-phase bacteria are also more susceptible to tobramycin-bicarbonate combinations than are biofilm bacteria. Taken together, these findings suggest that the antagonism found for bicarbonate+tobramycin combinations against biofilms (Figure 4) is likely multifactorial in origin. In addition to stationaryphase cells, biofilms also have phenotypically specific characteristics such as the extra-bacterial polymer+protein matrix and acidified interiors. These may act to turn additivity, against stationary-phase bacteria, into antagonism, against biofilms.

\section{Response-surface analysis}

The contrast between exponentially growing planktonic, stationary-phase planktonic and biofilm results highlights the complexity of this system and points up the need for methods of analysis that can quantitatively describe variable behaviour over a broad parameter-space. Isobolograms are a standard and widely used approach to examine drug interactions. ${ }^{28}$ Although they offer valuable insights, they do have limitations:

(1) The actual MIC value of an agent or combination of agents may be overestimated when testing using serial dilutions. This results in an underestimation of synergy.

(2) Plotting isobolograms from the growth-no growth interface implicitly neglects the potential therapeutic benefits of a partial inhibitory effect (less than MIC). 

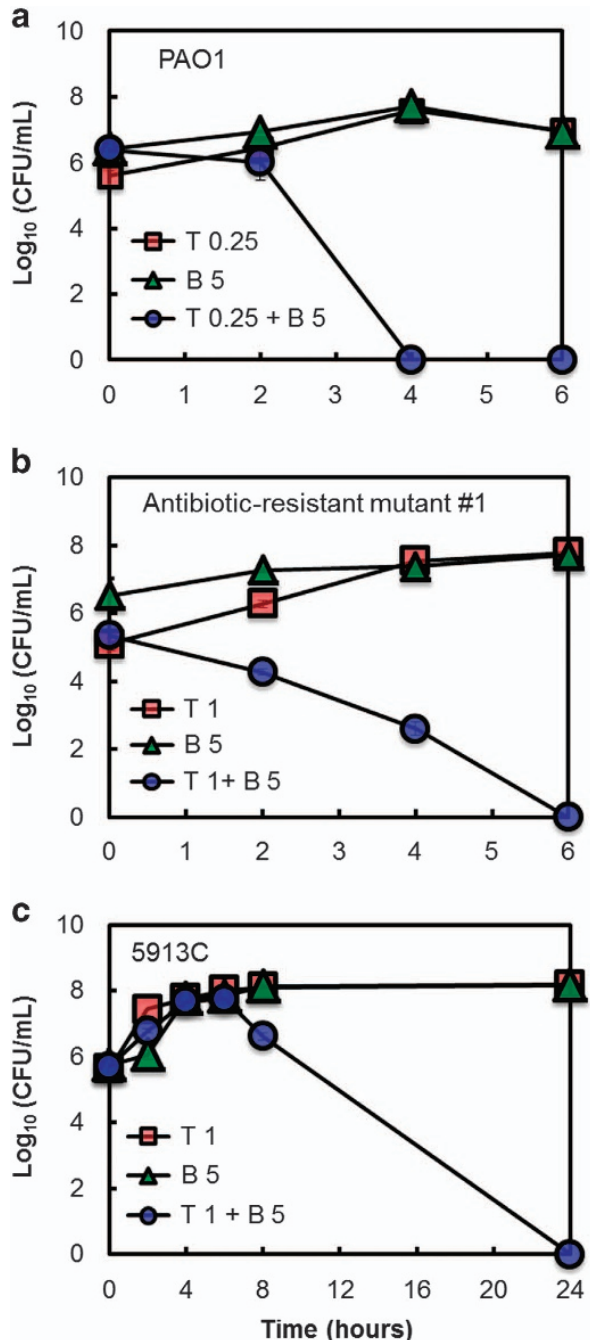

Figure 3. Time-kill assays demonstrating the synergy between bicarbonate and tobramycin against $P$. aeruginosa strains (a) PAO1, (b) antibiotic-resistant PA14 mutant \#1 and (c) 5913C. Concentrations of tobramycin and bicarbonate tested represent the combinations that produced the lowest $\Sigma$ FIC value. For strain PAO1, $0.25 \mu \mathrm{g} / \mathrm{ml}$ tobramycin and $5 \mathrm{mmol}$ bicarbonate; for antibioticresistant PA14 mutant \#1, $1 \mu \mathrm{g} / \mathrm{ml}$ tobramycin and $5 \mathrm{mmol}$ bicarbonate; and for strain 5913C, $1 \mu \mathrm{g} / \mathrm{ml}$ tobramycin and $5 \mathrm{mmol}$ bicarbonate were tested alone and in combination. Error bars represent s.e.m. (in some cases error bars are smaller than symbols). $N=3$.

To examine these drug combinations in greater detail than traditional methods, we develop a method of response-surface analysis. Using second-order polynomial interpolation of the checkerboard assays, we construct analytical dose-response surfaces that approximate the complex nature of nonlinear drug interactions with far greater resolution and nuance than allowed by traditional isobolograms. Measured response surfaces for planktonic and biofilm PAO1, 3470C, 4219D and 3470C are shown in Figure 5. The concave-upward response surfaces characterising the planktonic bacteria indicate synergy between bicarbonate and tobramycin; the concave-downward response surfaces characterising the biofilms indicate antagonism.

Furthermore, we use the measured effects of both tobramycin alone and bicarbonate alone to calculate surfaces describing Loewe additivity, as detailed in Materials and Methods. These surfaces are shown in Supplementary Figures S19-S21. The contrast with the measured response surfaces (Figure 5) is striking and consistent with our finding that neither planktonic nor biofilm bacteria respond additively to a treatment by a combination of tobramycin and bicarbonate.

To quantify the departure from additivity, and thereby quantify the degree of synergy or antagonism with the combination, we calculate difference surfaces, which measure the change in response from that expected from Loewe additivity, taking into account measurement error. By our construction, synergy will be indicated by a negative height and antagonism will be indicated by a positive height. Calculated difference surfaces (Figure 6) show, as expected, mostly negative heights for planktonic bacteria and mostly positive heights for biofilm bacteria. Response surface analysis of stationary-phase planktonic bacteria (Supplementary Figure S22) indicates a combination of synergy, additivity and antagonism.

These surfaces are notably rugged, occasionally extending above the zero plane for planktonic bacteria and below the zero plane for biofilms. This complex response is potentially clinically relevant, since drug levels are not expected to be homogenous throughout the airways and infection site in CF. This is highlighted by studies that have conducted quantitative aerosol deposition studies in patients with lung disease and shown non-uniform deposition. ${ }^{29}$ Thus, it is expected that the concentration of each administered drug will vary both with location in the body and with time. Accordingly, an assessment of the net effect of a combination over a given range of concentrations can simplify the complexity of the response surface and indicate whether a net benefit or a net harm is likely to occur. We provide such an assessment by integrating over the measured range of tobramycin and bicarbonate values-for PAO1 planktonic bacteria, this integral has a value of $-4.5 \pm 0.6$, indicating a net synergy, and for PAO1 biofilm bacteria, this integral has a value of $+8.50 \pm 5.61$, indicating a net antagonism. For stationary-phase PAO1 planktonic bacteria, this integral has a value of $-0.4 \pm 5.3$, so the net effect is nearly purely additive and is well resolved from the biofilm-state value of $+8.50 \pm 5.61$ (Supplementary Figure S22). This is in agreement with our isobologram analysis (Supplementary Figure S15) and with our inference that antagonism against biofilms likely results both from the presence of a large number of stationary-phase cells and characteristics specific to the biofilm phenotype. Notably, for the clinical isolate 3470C, the corresponding values are $-0.4 \pm 0.2$ for planktonic bacteria and $10.28 \pm 12.92$ for biofilm bacteria. This indicates that overall antagonism is likely stronger for $3470 \mathrm{C}$ biofilms than for PAO1 and that the overall synergy against $3470 \mathrm{C}$ planktonic bacteria is negligible. Finally, the difference surface for planktonic 4219D bacteria ranges between -50 and $+50 \%$, and the integral of this surface over the region shown is $-10.5 \pm 1$, indicating synergy between tobramycin and bicarbonate that is greater than that for PAO1 planktonic bacteria. However, for the 4219D biofilm, the difference surface is positive at most locations and the integral of the surface shown is $+67 \pm \sim 13$ (this uncertainty is from the ad hoc interpolation method used to create the response surface for the 4219D biofilm, because this biofilm was very poorly described by the Hill function used for all other systems). This indicates that the combination of tobramycin and bicarbonate against this biofilm has the strongest overall antagonism of all the systems we measure.

It is worth noting that this estimation of antagonism deliberately neglects the increased biofilm viability when bicarbonate is used without tobramycin and bicarbonate concentration is in the 100-300 mmol range. Therefore, this estimation of high antagonism substantially underestimates the net benefit to this biofilm of bicarbonate treatment.

\section{DISCUSSION}

Our findings indicate the need for caution when combining tobramycin and bicarbonate in CF treatment. 

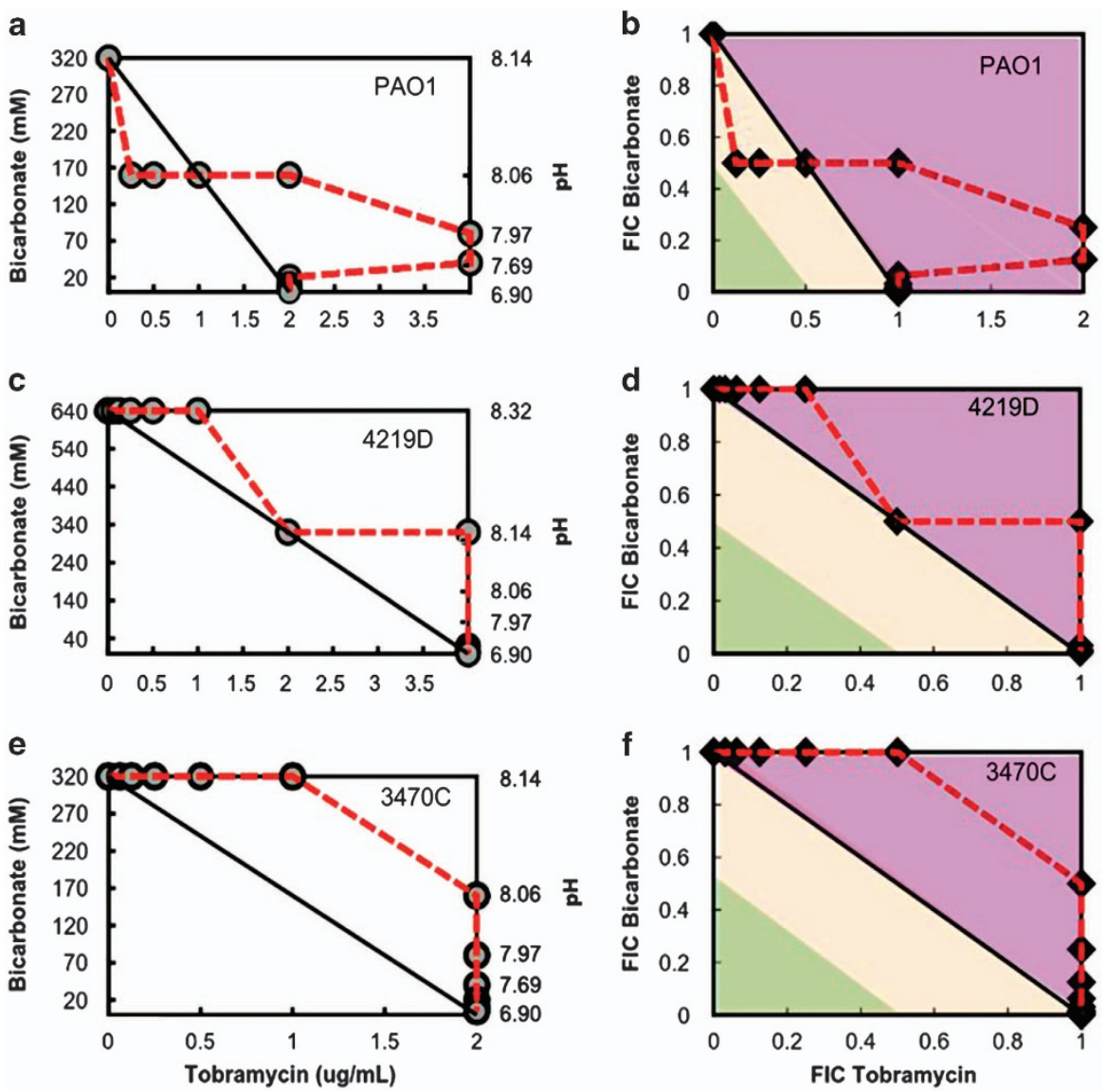

Figure 4. Isobologram analysis for biofilm cells of $P$. aeruginosa (a, b) PAO1, (c, d) 4219D and (e, f) 3470C. An additive-antagonistic effect is observed for the combination of tobramycin and bicarbonate against biofilm cells, in contrast to the strong synergistic effect observed with planktonic cells. Points along the isobologram represent the growth-no growth surface. (b, d, f) The orange shaded area represents the additive region, the purple shaded area represents the antagonistic region, and the green-shaded area represents the region of synergy.

If the expected distributions of each active agent in the body are known, the integral-based metric described above could be refined by limiting the range of concentrations over which it is performed and by rescaling the concentration axes to correspond to nonlinear probability distributions. Thus, development of new therapeutic approaches such as these studied here need to determine not just the nominal and administered doses, but also anticipate the effects of the local microenvironment and distributions within the infection site. Coupled with the data analysis described above, appropriate doses of the combination therapies can be determined.

\section{CONCLUSIONS}

Our results demonstrate that the combination of tobramycin and bicarbonate shows a strong synergistic effect against planktonic $P$. aeruginosa cells. As expected with a synergistic combination, addition of bicarbonate reduced the concentration of tobramycin needed to eradicate planktonic cells; it also significantly enhanced the rate of killing of cells in comparison with inhibitory concentrations of tobramycin alone. This opens the possibility of an additional role for bicarbonate therapy in CF patients, where it could augment the activity of tobramycin therapy against planktonic cells and early $P$. aeruginosa infections. However, the combination of tobramycin and bicarbonate was at best additive against biofilm cells of $P$. aeruginosa, and was in fact antagonistic in most combinations. Since inhaled tobramycin is standard-ofcare for CF patients infected with $P$. aeruginosa, and since chronic CF infections are widely thought to be caused by biofilms, our work indicates the need for caution in development of bicarbonate into a CF therapy.

This approach could also hold potential in the management of non-CF infections such as sepsis, urinary tract infections and meningitis. These approaches could also hold promise in the management of acute burn and post-surgical wounds, but it is clear that more work needs to be done to fully understand the effects of local $\mathrm{pH}$ at wound infection sites. ${ }^{30}$

Finally, we present an improved method for analysing drug interactions using analytic dose-response surfaces. These allow more accurate and more nuanced estimation of regimes of synergy and antagonism than do standard isobolograms, which have limited analytical and predictive power. Continuous response surfaces have improved potential to find untested synergistic combinations. By combining measured response surfaces with predictions based on Loewe additivity theory, we can consolidate information on the effect of a wide range of combined concentrations into a single number that measures the net effect, both synergistic and additive. This can simplify complex response surfaces to allow rapid initial estimation of the potential benefit or harm of a therapeutic combination. Further information and annotated code is made available in Supplementary Information as a resource for the community.

\section{MATERIALS AND METHODS}

Bacterial strains and growth conditions

$P$. aeruginosa strains used include wild-type ${ }^{31}$ laboratory strains PA14 and PAO1 and clinical isolates from patients with $\mathrm{CF}^{32}$ (gift from Marvin 
a

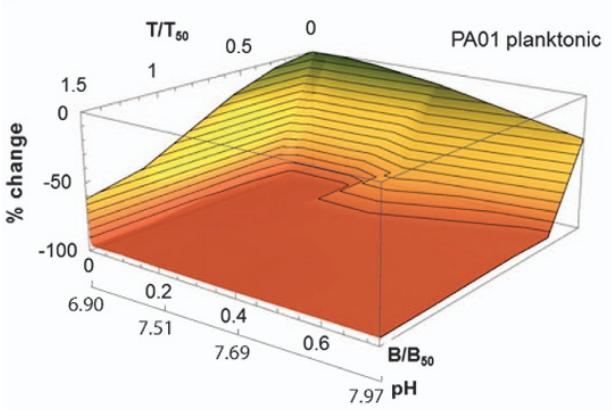

C

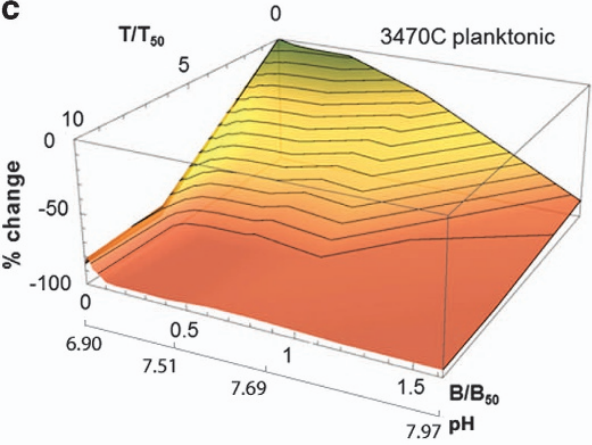

e

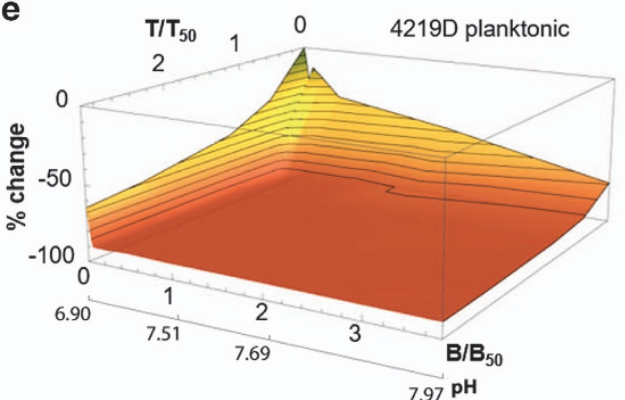

b

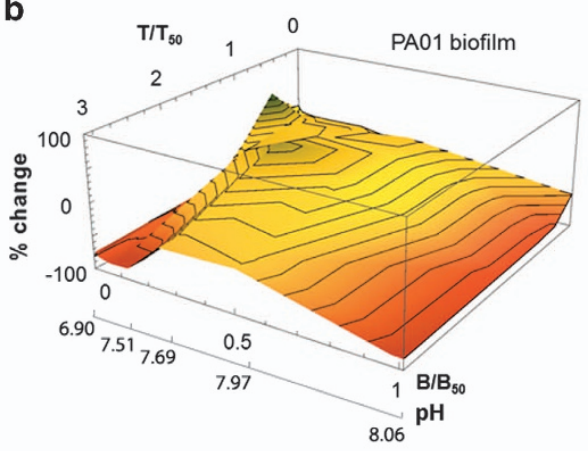

d

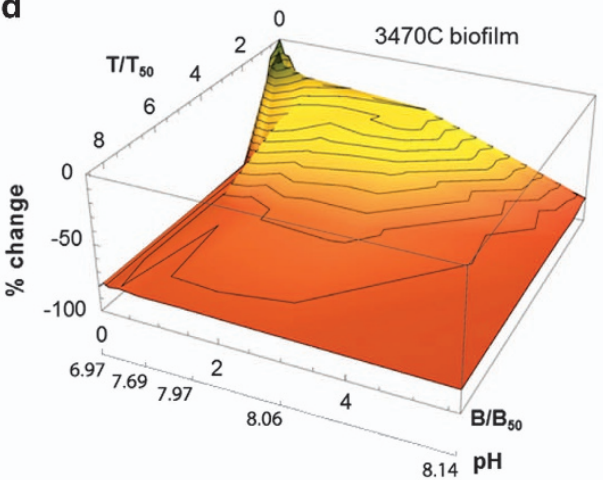

f

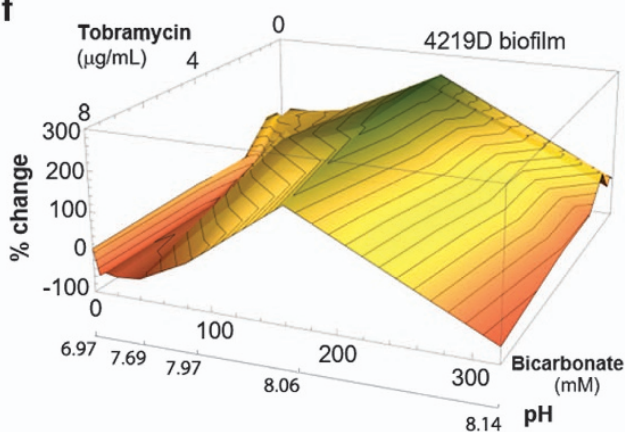

Figure 5. Measured response surfaces for tobramycin and bicarbonate treatments of (a, c, e) planktonic bacteria and (b, d, $\mathbf{f})$ biofilms. Contour lines show increments of $10 \%$ change. (a-e) Tobramycin and bicarbonate concentrations are plotted as the fraction of the concentration that produces $50 \%$ growth inhibition. (f) For the 4219D biofilm, the one-dimensional curves along the bicarbonate axis (with no tobramycin) and along the tobramycin axis (with no bicarbonate) are non-monotonic. This prevents determination of 50\% growth inhibition values and therefore actual concentrations are used instead. (a, e) For PAO1 and 4219D, the planktonic response surface is steeply concave upward, reflecting synergy between tobramycin and bicarbonate for planktonic bacteria. (c) For 3470C, the response surface is only shallowly curved, indicating little overall synergy for planktonic bacteria. (b, d, f) For all strains, the biofilm response surface is concave downward. This reflects synergy between tobramycin and bicarbonate for planktonic bacteria and antagonism between tobramycin and bicarbonate for biofilm bacteria. (b, f) For PAO1 and 4219D, portions of the biofilm response surface extend above $0 \%$ change, indicating that combination of tobramycin and bicarbonate can enhance viability over that of the untreated biofilm.

Whiteley, UT Austin). To generate spontaneous antibiotic-resistant mutants, WT PA14 cultures, were grown overnight in antibiotic-free media, and plated on tobramycin $8 \mu \mathrm{g} / \mathrm{ml}$ agar. Antibiotic-resistant mutants grew colonies and were archived in $20-30 \%$ glycerol at $-80^{\circ} \mathrm{C}$. Of the four independent antibiotic-resistant mutants (strains 1-4) used in this study, strain 1 was used in our previous work, which examined the effect of population spatial structure and metabolism on antibiotic resistance. ${ }^{15}$ As part of this previous work, ${ }^{15}$ this strain was sequenced (Illumina MiSeq) and sequence information is deposited in the NCBI Short-Read Archive (accession no. SRP042054). Three single-nucleotide polymorphisms (SNPs) unique to this strain were identified, in genes TrkH (potassium uptake membrane protein), EF-Tu (elongation factor) and PhzD (phenazine biosynthesis protein); each of these mutations is a plausible candidate, alone or in combination, to confer aminoglycoside resistance. ${ }^{15}$ Elongation factor Tu (EF-Tu) has a crucial role in the elongation phase of bacterial protein synthesis, in which it delivers aminoacylated tRNAs (aa-tRNAs) to the ribosome. ${ }^{33}$ Aminoglycoside antibiotics inhibit bacterial protein synthesis by targeting the ribosome. ${ }^{34}$ Mutations in the eukaryotic elongation factor 1 (EF-1), analogous to bacterial EF-Tu, and other bacterial elongation factors, such as EF-G, have been associated with aminoglycoside resistance, ${ }^{35}$ indicating the possibility that the EF-Tu mutation. TrkH is a hydrophobic, membrane protein and a constituent of the potassium uptake system, which mediates the symport of potassium and hydrogen ions. Mutations in Trk system have been associated with altered aminoglycoside susceptibility. The amino-acid residue affected by one of these mutations was close to the ion channel, possible leading to increased proton influx and diminished membrane potential. ${ }^{35}$ Aminoglycosides require the membrane potential component of the proton motive force for active cellular uptake ${ }^{36}$ and adaptation to this class of antibiotics frequently proceeds through mutations that diminish the generation of proton motive force. The $p h z D 2$ gene is part of the phenazine biosynthetic cluster, encoded by two redundant operons phzA1-G1 and phzA2-G2. ${ }^{37}$ 

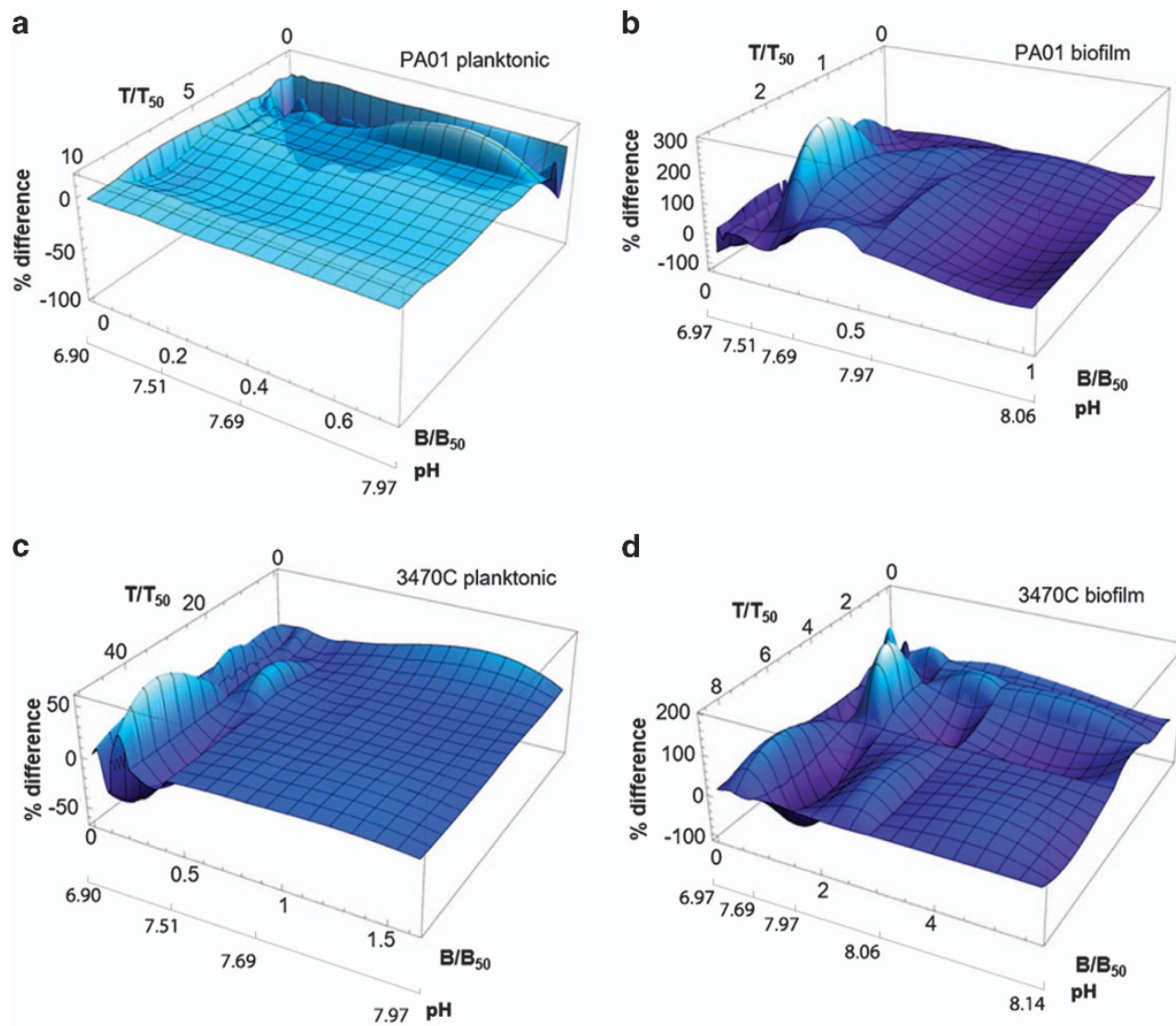

d
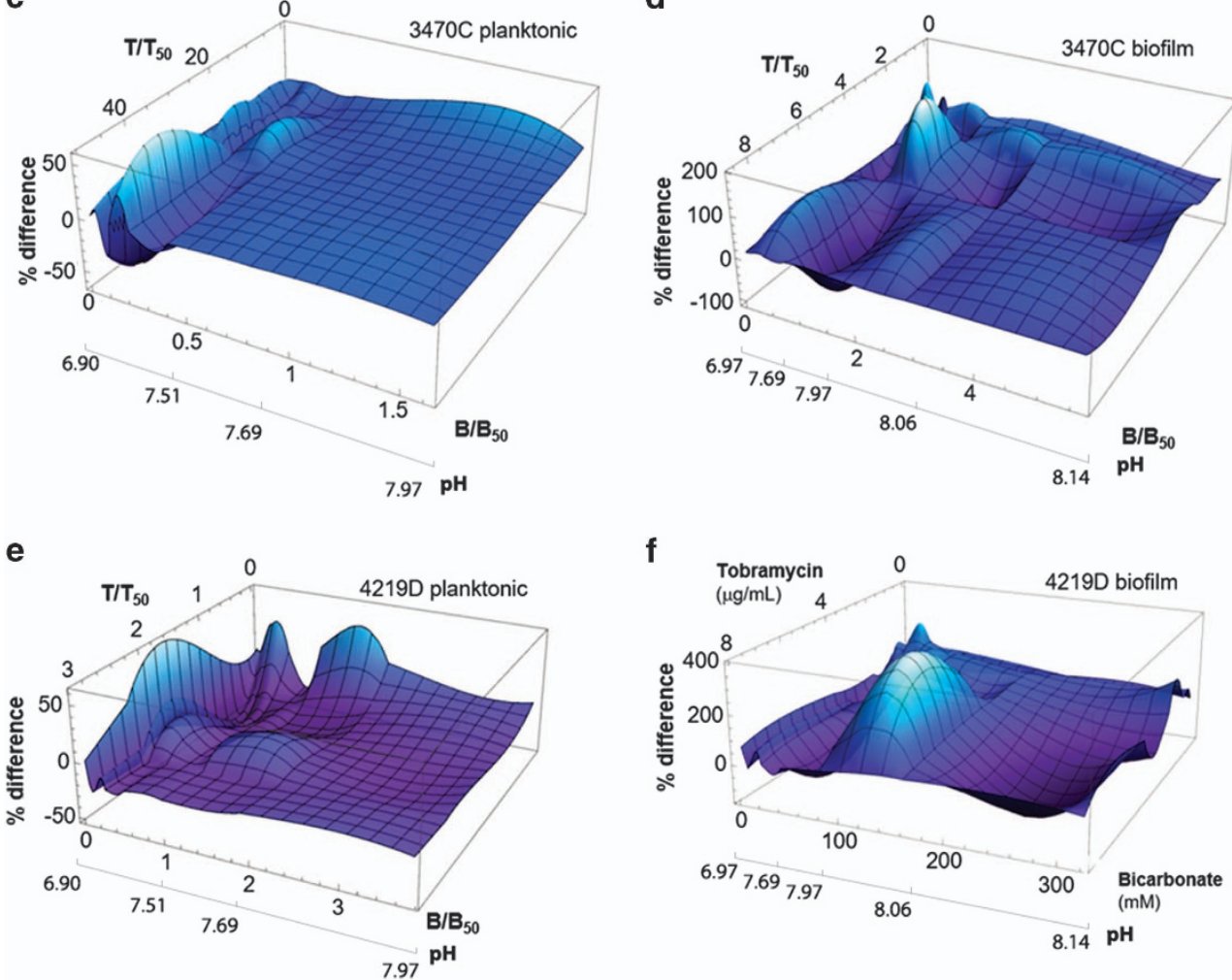

Figure 6. Difference surfaces measure the departure of measured response from that expected from Loewe additivity for (a, c, e) planktonic bacteria and (b, d) biofilms. (f) For 4219D biofilms, we measure the departure from a strictly additive surface. By our construction, a negative value for \% difference indicates a synergistic interaction between tobramycin and bicarbonate at the corresponding combination, and a positive height indicates an antagonistic interaction. (a) The difference surface for planktonic PAO1 is consistently negative within the $0 \%$ difference border, indicating that the interaction between tobramycin and bicarbonate is everywhere synergistic. This surface is rendered semi-transparent for better visualisation. (b) The difference surface for biofilm PAO1 is positive at most locations, indicating overall antagonism, and negative at a few locations, indicating that within that limited parameter space the tobramycin-bicarbonate interaction is synergistic against the biofilm. (c) The difference surface for planktonic $3470 \mathrm{C}$ bacteria ranges between -50 and $+50 \%$, and the integral of this surface over the region shown is close to zero. This indicates that the net synergy between tobramycin and bicarbonate is negligible when averaged over the combinations measured. (d) The difference surface for biofilm $3470 \mathrm{C}$ is positive at most locations, indicating overall antagonism, and negative at a few locations, indicating that within that limited parameter space the tobramycin-bicarbonate interaction is synergistic against the biofilm. (e) The difference surface for planktonic 4219D bacteria ranges between -50 and $+50 \%$, and the integral of this surface over the region shown is -3.9 , indicating overall net synergy between tobramycin and bicarbonate. (f) For the biofilm, the difference surface for biofilm 4219D is positive at most locations and the integral over the area shown is +67, indicating that the combination of tobramycin and bicarbonate against this biofilm has the strongest overall antagonism of all the systems we measure.

To the best of our knowledge, there is no direct evidence of $p h z D$ mutations conferring aminoglycoside resistance, and the redundant nature of this gene makes its role further unlikely.

The clinical CF isolates used in this work, have been used in previous work, ${ }^{32}$ and have been subject to transcriptome analysis. This determined that these strains had been acquired a range of adaptive traits during their evolution in the in vivo host. The MIC for tobramycin for the clinical
CF isolates was tested and reported in Supplementary Table S1 of our previous work. ${ }^{15}$ These MIC values are (in $\mu \mathrm{g} / \mathrm{ml}$ ) 0.07 for strain $3640 \mathrm{D}, 0.3$ for strains 4219D, 0476M, 0.6 for strains 3470C, 3639M, 4278M, 4218C, 0324C, 2159M, 4220M, 5912M, 1.2 for strains 2773C, 1913C, 3488D, 2.4 for strains $5623 \mathrm{M}, 5914 \mathrm{M}$ and 4.8 for $5913 \mathrm{C}$. In this nomenclature, the terminal ' $C$ ' indicates a classic colony phenotype, the terminal ' $M$ ' indicates a mucoid (alginate overproducing) colony phenotype, and the terminal ' $D$ ' 
indicates a dwarf, or small-colony, phenotype. Small-colony phenotypes are frequently associated with increased production of the non-alginate extracellular polysaccharides PsI and/or Pel. Increases in the production of alginate, Psl and Pel have been linked to increased tobramycin resistance for biofilms. ${ }^{38-40}$

All bacterial strains were grown in Luria-Bertani broth or on LB agar ${ }^{41}$ except where otherwise indicated. Overnight cultures were shaken at 180 r.p.m. for $16-18 \mathrm{~h}$ at $37^{\circ} \mathrm{C}$.

\section{Antibiotics}

Tobramycin (Indofine Chemical Company, Hillsborough, NJ, USA) and sodium bicarbonate (Thermo Fisher Scientific, Waltham, MA, USA) were obtained as standard powders. A stock solution of $50 \mathrm{mg} / \mathrm{ml}$ tobramycin was stored at $4{ }^{\circ} \mathrm{C}$ prior to use. The desired concentration of sodium bicarbonate solution was freshly prepared prior to use for each experiment.

\section{Minimum inhibitory concentration determination for} planktonic cells

MICs to tobramycin and bicarbonate were measured using broth microdilution methods. MICs were determined by observing visual turbidity and measuring optical density (OD) at $600 \mathrm{~nm}$. The lowest concentration that inhibited visual growth, which corresponded to $90 \%$ inhibition of bacterial growth (reported as MIC), was used for further analysis, except where stated otherwise for undiluted, dense planktonic suspensions.

\section{Checkerboard assay to study drug interactions against planktonic} $P$. aeruginosa cells

Standard checkerboard microdilution assays ${ }^{42,43}$ were used to test the combined antimicrobial activity of tobramycin and bicarbonate against planktonic cells of different $P$. aeruginosa strains. In brief, an $8 \times 8$ array of serial twofold dilutions of the two agents were mixed together in a flat-bottom, 96-well microtiter plate (polystyrene) such that each row (or column) contained a fixed amount of one agent and increasing amounts of the second agent. This resulted in a total of 64 different combinations. For each assay, the serial dilutions of each individual agent were tested alone (to measure the $\mathrm{MIC}$ ), and control wells containing untreated cells were also grown. Bacteria were grown overnight in LB medium (18-24 $\mathrm{h}$, resulting in stationary phase cultures), and the next day $\sim 10^{5}$ cells from these cultures were added to each well. Each strain was tested in duplicate. Plates were sealed with parafilm and incubated at $37^{\circ} \mathrm{C}$ under static conditions. After overnight incubation (16-18 h), optical density was measured at $600 \mathrm{~nm}\left(\mathrm{OD}_{600}\right)$. An example of such measurements is given in Supplementary Figure S1A. The FIC was calculated for each well along the growth-no growth interface (corresponding to 90\% inhibition in the presence of the combination, with each agent below its own MIC). For agents $A$ and $B$, the FIC of the combination is calculated $\mathrm{as}^{23}$

$$
\Sigma \mathrm{FIC}_{\mathrm{A}+\mathrm{B}}=\mathrm{FIC}_{\mathrm{A}}+\mathrm{FIC}_{\mathrm{B}} \text {, whereFIC } \mathrm{A}=\frac{\mathrm{MIC}_{\mathrm{A}+\mathrm{B}}}{\mathrm{MIC}_{\mathrm{A}}} \text { and } \mathrm{FIC}_{\mathrm{B}}=\frac{\mathrm{MIC}_{\mathrm{A}+\mathrm{B}}}{\mathrm{MIC}_{\mathrm{B}}}
$$

An example of calculated $\Sigma F I C$ indices is given Supplementary Figure S1B. The $\Sigma$ FIC index was interpreted as follows: $\Sigma F I C \leqslant 0.5$ indicates a synergistic interaction; $\geqslant 0.5$ and $<1$ indicates an additive effect; and $>1$ indicates antagonism.

The results of the checkerboard assays were represented graphically using isobolograms. Combinations that fall along the line connecting the MIC values of the two agents (line of additivity) are additive interactions. If the combination is synergistic, the isobol will be overall concave-up. For combinations that are antagonistic, the isobol will be overall concave-down. Supplementary Figure S2 labels additive, synergistic and antagonistic regions on a sample coordinate system. Further, the magnitude of the overall average curvature indicates the degree of synergy or antagonism, respectively.

\section{Time-kill assays}

To examine the rate of killing of the synergistic combination, time-kill assays were performed. Briefly, 96-well microtiter plates were set up using synergistic combinations (from the checkerboard assays), with concentrations of the individual agents alone, and untreated cells as controls. Bacteria $\left(\sim 10^{5}\right.$ cells) were added to each well. Plates were incubated at $37^{\circ} \mathrm{C}$ for $24 \mathrm{~h}$ under static conditions. Colony-forming units per $\mathrm{ml}$
(CFU/ml) were counted at $0,2,4,6,8$ and $24 \mathrm{~h}$ after beginning treatment. To count the number of colonies, serial dilutions of the bacterial suspension were plated on LB agar. Plates were incubated at $37^{\circ} \mathrm{C}$ overnight (16-18 h) and CFUs were counted. For each strain tested, assays were performed in duplicate. Each combination or concentration at a given time point was tested in duplicate.

\section{Measurement of $\mathrm{pH}$}

Different concentrations of bicarbonate were added to sterile LB media and the $\mathrm{pH}$ was measured using a $\mathrm{pH}$ meter (Thermo Scientific Orion 2 Star). Prior to use, the instrument was calibrated using standard solutions of $\mathrm{pH} 4,7$ and 10.

Testing the effect of the tobramycin+bicarbonate combination against preformed biofilms

Biofilms were grown in round-bottom, untreated, 96-well microtiter plates as previously described. ${ }^{44,45}$ Briefly, bacterial $\left(\sim 10^{5}\right.$ cells) were added to well of the microtiter plate in an $8 \times 8$ array to test different combinations of tobramycin and bicarbonate. In addition, wells to test serial dilutions of each agent alone, and control wells for untreated cells were also set up. Each strain was tested in duplicate. Plates were sealed with parafilm and incubated at $37^{\circ} \mathrm{C}$ under static conditions for $18-24 \mathrm{~h}$ (overnight). The next day, wells were washed twice with sterile LB medium. Different combinations of tobramycin and bicarbonate (dissolved in LB medium) were added to the $8 \times 8$ array, such that each row (or column) contained a fixed amount of one agent and increasing amounts of the second agent (total volume $150 \mu \mathrm{l}$ ). To obtain the minimum biofilm inhibitory concentration, serial dilutions of each agent were also tested alone (total volume $150 \mu \mathrm{l}$ ). The control wells were replaced with sterile LB medium without any antimicrobial agent. The plates were sealed with parafilm and incubated at $37^{\circ} \mathrm{C}$ static for $18-24 \mathrm{~h}$ (overnight). The next day, the wells were washed with sterile LB medium twice and effect of the treatment on biofilm cells was assessed with crystal violet staining and the XTT assay. ${ }^{45,46}$

\section{Crystal violet assay for biofilm mass}

The crystal violet assay was performed as previously described. ${ }^{45}$ Briefly, preformed biofilms (both treated and untreated controls) were washed twice with sterile LB medium. Cells were fixed at the bottom and sides of the wells by treating them with $100 \%$ methanol for 15 min. Following removal of methanol, $0.1 \%$ filter-sterilised crystal violet solution ${ }^{47}$ was added to the well and allowed to stain the biofilm for $30 \mathrm{~min}$. Wells were washed in water and the dye was solubilised using 100\% ethanol. Two-hundred microlitres of well contents were transferred to a new, clear, flat-bottom, 96-well plate and absorbance was read at $600 \mathrm{~nm}$.

\section{$\mathrm{XTT}$ assay for metabolic activity in biofilms}

The XTT assay was performed as previously described. ${ }^{46}$ Briefly, preformed biofilms (either treated or untreated) were washed twice with sterile LB medium. XTT was dissolved to make a stock solution of $1 \mathrm{mg} / \mathrm{ml}$. For Menadione, a $7 \mathrm{mg} / \mathrm{ml}$ stock solution was prepared in acetone and then diluted 1:100 in distilled water. A solution of LB: XTT: Menadione (79:20:1) was prepared. The solution of LB: XTT: Menadione (200 $\mu \mathrm{l})$ was added to each well, and the plates were incubated at $37^{\circ} \mathrm{C}$ in the dark (sealed with aluminum foil) for $4 \mathrm{~h}$. One-hundred-fifty microlitres of well contents were transferred to a new, clear, flat-bottom, 96-well plate and absorbance was read at $492 \mathrm{~nm}$.

\section{Screen for biofilm formation by $P$. aeruginosa strains}

The laboratory strain PAO1 and 17 clinical CF isolates (mucoid, dwarf and classic phenotype), ${ }^{32}$ were screened for biofilm formation using the microtiter dish biofilm assay and quantified using the crystal violet and $\mathrm{XTT}$ methods. Using the crystal violet assay, clinical strains $3639 \mathrm{M}, 4278 \mathrm{M}$, 3470C, 1913C, 0476M, 4220M, 4219D and laboratory strain PAO1 show robust biofilm formation (Supplementary Figure S3A). Crystal violet is a basic dye that binds to negatively charged surface molecules. It thus stains the extracellular matrix, live and dead cells of the biofilm.

To quantify changes in metabolic activity, we use the XTT assay. In metabolically active cells, XTT is reduced to a water-soluble formazan derivative that can be quantified colorimetrically. Strains 3639M, 4278M, 3470C, 1913C, 0476M, 4220M, 4219D and PAO1 that showed robust 
biofilm formation in the crystal violet assay also demonstrated the presence of live, metabolically active cells in the XTT assay (Supplementary Figure S3B). On the basis of the results of this screen, three strains, PAO1, 4219D and 3470C, were chosen for testing the effects of the tobramycinbicarbonate combination on biofilms.

Measurement of the effect of tobramycin+bicarbonate on biofilms This testing was done using the XTT assay. The XTT assay is a measure of metabolic activity, and therefore its readout is a proxy measurement for metabolic activity of bacterial cells. Preformed biofilms (treated or untreated with tobramycin and bicarbonate) were treated with XTT and absorbance was measured as the readout. An increase in XTT absorbance can indicate greater metabolic activity and/or more active cells, and a decrease in XTT absorbance can indicates decreased metabolic activity (i.e., bacteria entering a more-inactive state) and/or killing of cells, but cannot differentiate between the two.

Measurement of the effect of tobramycin+bicarbonate on high density, stationary phase cells

Briefly, a population of $P$. aeruginosa PAO1 cells was grown to an $O D=2$, to represent stationary phase cells. Different combinations of tobramycin and bicarbonate were made in medium containing salt+water $(1 \%$ sodium chloride in water) to represent the salinity of standard Luria-Bertani medium, but with no nutrients. This was intended to prevent activation of cell metabolism to planktonic phase as would occur in the presence of medium containing fresh nutrients. Stationary-phase cells $(O D=2)$ were added to these different combinations of tobramycin+bicarbonate in salt water, in a 96-well flat-bottom plate (as used previously). Plates were incubated at $37^{\circ} \mathrm{C}$ shaking overnight and $\mathrm{OD}_{600}$ values were read after 16-18 h. Owing to the high density of cells, the lowest concentration that inhibited growth corresponded to $\sim 50 \%$ inhibition of bacterial growth (reported as $\mathrm{MIC}_{50}$ ). Therefore, $\mathrm{MIC}_{50}$ was used for further analysis including isobolograms, FIC plots and response surface.

\section{Response surface analysis}

To construct and analyse response surfaces, we used Mathematica and Google Sheets. Data from checkerboard assays were arranged into arrays ('PercentChange[T,B]') giving the measured percent change in optical density (for planktonic cells) or XTT metabolic readout (for biofilms), compared with untreated bacteria. Estimated measurement errors were also arranged into arrays ('Error[T,B]') for each checkerboard assay. For each two-dimensional array, the tobramycin concentration was mapped to the first, ' $x$ ' position index and the bicarbonate concentration was mapped to the second, ' $y$ ' position index. Arrays were imported into Mathematica.

Calculating the surface describing loewe additivity. Loewe additivity is a common reference model to define drug interactions. ${ }^{25}$ According to this model, the combined effect of two drugs is predicted from the sum of the effects of the individual components. For both tobramycin alone and bicarbonate alone, we assumed Hill kinetics of a variable ligand concentration, [L], binding randomly to a finite number of receptors with disassociation constant $K_{\mathrm{d}}$. The resulting Hill equation has the form

$$
H([L])=\frac{[L]^{m}}{K_{\mathrm{d}}^{m}+[L]^{m}}
$$

where $m$ measures the self-cooperativity of the ligand and the slope of the linear regime of the sigmoidal curve $H([L])$. By normalising ligand concentration to the concentration that produces $50 \%$ growth inhibition, we remove $K_{d}^{m}$ from the expression, which we can also write in terms of the maximum measured value, $H_{\max }$ and the minimum measured value, $H_{0}$ :

$$
H([A])=\left(H_{\max }-H_{0}\right) \frac{\left([A] / A_{50}\right)^{m}}{\left[1+\left([A] / A_{50}\right)^{m}\right]}+H_{0}
$$

Here, $H_{0}$ corresponds to the absence of any treatment, and $H_{\max }$ corresponds to the greatest reduction in growth or viability.

We used Mathematica to fit this function to each of the one-dimensional data sets describing the response to increasing concentrations of tobramycin (without bicarbonate) and to increasing concentrations of bicarbonate (without tobramycin), thus:

These fits (Supplementary Figure S4 for planktonic cells and Supplementary Figure S5 for biofilms) were used to determine the concentration that produces $50 \%$ growth inhibition for tobramycin alone $\left(T_{50}\right)$ and for bicarbonate alone $\left(B_{50}\right)$, and the $m$ values for tobramycin alone $\left(m_{\mathrm{T}}\right)$ and for bicarbonate alone Using these parameters, we calculated and plotted the surface-describing Loewe additivity as defined in Meletidias et $a{ }^{25}$ In brief, weighting coefficients are functions of tobramycin concentration $[T]$ and bicarbonate concentration $[B]$, thus:

$$
\text { Weight }_{\text {Tob }}=\left(\frac{[T]}{T_{50}}\right) /\left(\frac{[T]}{T_{50}}+\frac{[B]}{B_{50}}\right)
$$

and

$$
\text { Weight }_{\text {Bicarb }}=\left(\frac{[B]}{B_{50}}\right) /\left(\frac{[B]}{B_{50}}+\frac{[T]}{T_{50}}\right) \text {. }
$$

These weights are used to calculate the local slope of the surface,

$$
m_{\text {local }}=\left(\text { Weight }_{\text {Tob }}\right) m_{T}+\left(\text { Weight }_{\text {Bicarb }}\right)\left(m_{B}\right),
$$

and to weight the local contributions of each $50 \%$ growth inhibition value value,

$$
U_{0}=\left(\left(T_{50}\right)^{\left(\text {Weight }_{\mathrm{Tob}}\right)}\right)\left(\left(B_{50}\right)^{\left(\text {Weight }_{\text {Bicarb }}\right)}\right)
$$

The total number of $50 \%$ growth inhibition units, also called 'potency units,' from both tobramycin and bicarbonate, is given by

$$
U=\frac{[T]}{T_{50}}+\frac{[B]}{B_{50}}
$$

The Loewe additivity surface is a function of the number of potency units, the local slope, and the weighted local contributions to $50 \%$ growth inhibition, thus:

$$
\begin{aligned}
& \text { Loewe }=(\text { Lower Bound }- \text { Upper Bound }) \frac{\left(U / U_{0}\right)^{m_{\text {local }}}}{1+\left(U / U_{0}\right)^{m_{\text {local }}}} \\
& \quad+\text { Lower Bound }
\end{aligned}
$$

where LowerBound is the lowest value measured for growth or viability, and UpperBound is the growth or viability value measured without treatment. Thus, Equation (8) has the same form as the Hill function in Equation (2), but has now been generalised to describe a two-dimensional parameter space.

Our step-by-step approach to calculating the Loewe surface is shown in the annotated Mathematica notebook in Supplementary Material.

The Loewe surface presented a challenge for numerical analysis, as its limits are not well defined as they approach zero. We handled this by redefining our zero values to 0.0001 for Bicarbonate and Tobramycin. The resulting Loewe function was used to populate an array ('Loewe[T,B]') describing the response predicted by Loewe additivity.

Calculating the surface describing the degree of synergy or antagonism. The departure of the measured response ('PercentChange') from that calculated from Loewe additivity theory ('Loewe') indicates that the effect of a combination is synergistic or antagonistic, rather than additive. To this difference, we added the measurement errors ('Error') to establish an upper bound on the difference surface-i.e., to create a surface describing the upper bound. We then created a surface describing the lower bound by subtracting the measurement errors ('Error') from the difference. The calculated difference surfaces were thus given by

Upper Difference $[\mathrm{T}, \mathrm{B}]=$ Loewe $[\mathrm{T}, \mathrm{B}]-$ PercentChange $[\mathrm{T}, \mathrm{B}]+\operatorname{Error}[\mathrm{T}, \mathrm{B}]$.

Lower Difference $[\mathrm{T}, \mathrm{B}]=$ Loewe $[\mathrm{T}, \mathrm{B}]$ - PercentChange $[\mathrm{T}, \mathrm{B}]$ - Error $[\mathrm{T}, \mathrm{B}]$. We interpolated Upper and Lower Difference $[T, B]$ in Mathematica to get an integrable form.

For $4219 \mathrm{D}$ biofilms, the response to increasing concentrations of tobramycin alone and of bicarbonate alone was non-monotonic. This prevented fitting of a Hill function, so the response surface was instead estimated as a strictly additive effect of tobramycin and bicarbonate at their respective concentrations with $20 \%$ uncertainty at all points.

Calculating net synergy or antagonism of a wide range of combinations. We numerically integrated the upper and lower difference surfaces and divided by the projected, planar area under the surfaces to give a quantitative upper and lower bound of synergy, additivity, or antagonism. We took the average of the two area-normalised integrals to give the net measure of tobramycin+bicarbonate interaction, and the difference between the mean and the bounding values as the uncertainty. Our definition of the difference surface means that a value of zero corresponds 
to no net departure from Loewe additivity, a negative value corresponds to a net synergistic effect, with the magnitude indicating the overall strength of the synergy, and a positive value corresponds to a net antagonistic effect, with the magnitude indicating the overall strength of the antagonism.

A Mathematica notebook giving the code, with explanatory comments, that was used for all parts of this work is included in Supplementary Material.

\section{ACKNOWLEDGEMENTS}

We thank Marvin Whiteley (The University of Texas at Austin) for his gift of CF clinical isolates and scientific discussions, Andreas Matouschek (The University of Texas at Austin) for use of his laboratory equipment and Kendra Rumbaugh (Texas Tech Health Sciences Center) for scientific input. We thank the Statistical Consulting Group (The University of Texas at Austin) and Biswanadham Sridhara and Dennis Wylie (bioinformatics consultants at The University of Texas at Austin) for discussion of response surface fitting and analysis, and Kanishk Jain for suggestions on fitting. This work was supported by start-up funds from The University of Texas at Austin to V.D.G., a gift from ExxonMobile to V.D.G., and a Microbiology Summer Merit Award from UT Austin to K.S.K. The funders had no role in the study design, data collection and interpretation, or the decision to submit the work for publication.

\section{CONTRIBUTIONS}

K.S.K. designed and performed the experiments, performed FIC and isobologram analysis and wrote the manuscript. J.S. did response-surface analysis and wrote the manuscript. O.S. developed approaches for response-surface analysis. H.S. designed experiments and wrote the manuscript. V.D.G. directed the research, designed experiments and analysis, and wrote the manuscript. V.D.G. and K.S.K. are guarantors for this work.

\section{COMPETING INTERESTS}

H.D.S. holds stock in and received an honorarium from Respira Therapeutics. The terms of this arrangement have been reviewed and approved by the University of Texas at Austin in accordance with its policy on objectivity in research. The remaining authors declare no conflict of interest.

\section{REFERENCES}

1. Levy, S. B. The 2000 Garrod lecture. Factors impacting on the problem of antibiotic resistance. J. Antimicrob. Chemother. 49, 25-30 (2002).

2. Hirsch, E. B. \& Tam, V. H. Impact of multidrug-resistant Pseudomonas aeruginosa infection on patient outcomes. Expert Rev. Pharmacoecon. Outcomes Res. 10, 441-451 (2010)

3. Bodey, G. P., Bolivar, R., Fainstein, V. \& Jadeja, L. Infections caused by Pseudomonas aeruginosa. Rev. Infect. Dis. 5, 279-313 (1983).

4. Turner, K. H., Everett, J., Trivedi, U., Rumbaugh, K. P. \& Whiteley, M. Requirements for Pseudomonas aeruginosa acute burn and chronic surgical wound infection. PLoS Genet. 10, e1004518 (2014).

5. Yahr, T. L. \& Greenberg, E. P. The genetic basis for the commitment to chronic versus acute infection in Pseudomonas aeruginosa. Mol. Cell 16, 497-498 (2004).

6. Serra, R. et al. Chronic wound infections: the role of Pseudomonas aeruginosa and Staphylococcus aureus. Expert Rev. Anti Infect. Ther. 13, 605-613 (2015).

7. Hart, C. A. \& Winstanley, C. Persistent and aggressive bacteria in the lungs of cystic fibrosis children. Br. Med. Bull. 61, 81-96 (2002).

8. Davies, J. C. Pseudomonas aeruginosa in cystic fibrosis: pathogenesis and persistence. Paediatr. Respir. Rev. 3, 128-134 (2002).

9. Drenkard, E. Antimicrobial resistance of Pseudomonas aeruginosa biofilms. Microbes Infect. 5, 1213-1219 (2003).

10. Fricks-Lima, J. et al. Differences in biofilm formation and antimicrobial resistance of Pseudomonas aeruginosa isolated from airways of mechanically ventilated patients and cystic fibrosis patients. Int. J. Antimicrob. Agents 37, 309-315 (2011).

11. Clark, S. T. et al. Phenotypic diversity within a Pseudomonas aeruginosa population infecting an adult with cystic fibrosis. Sci. Rep. 5, 10932 (2015).

12. Prayle, A. P. et al. The pharmacokinetics and toxicity of morning vs. evening tobramycin dosing for pulmonary exacerbations of cystic fibrosis: A randomised comparison. J. Cyst. Fibros. (e-pub ahead of print 15 August 2015; doi:10.1016/j. jcf.2015.07.012) (2015).

13. Prayle, A., Watson, A., Fortnum, H. \& Smyth, A. Side effects of aminoglycosides on the kidney, ear and balance in cystic fibrosis. Thorax 65, 654-658 (2010).

14. Bernier, S. P., Letoffe, S., Delepierre, M. \& Ghigo, J. M. Biogenic ammonia modifies antibiotic resistance at a distance in physically separated bacteria. Mol. Microbiol. 81, 705-716 (2011).
15. Kaushik, K. S., Ratnayeke, N., Katira, P. \& Gordon, V. D. The spatial profiles and metabolic capabilities of microbial populations impact the growth of antibiotic-resistant mutants. J. R. Soc. Interface 12, doi:10.1098/rsif.2015.0018 (2015).

16. Letoffe, S., Audrain, B., Bernier, S. P., Delepierre, M. \& Ghigo, J. M. Aerial exposure to the bacterial volatile compound trimethylamine modifies antibiotic resistance of physically separated bacteria by raising culture medium $\mathrm{pH}$. mBio 5, e00944-00913 (2014).

17. Hansson, G. C. Sparkling water--bicarbonate for cervix and cystic fibrosis. J. Physiol. 588, 2685 (2010).

18. Wine, J. J. Cystic fibrosis: the 'bicarbonate before chloride' hypothesis. Curr. Biol. 11, R463-R466 (2001).

19. Borowitz, D. CFTR, bicarbonate, and the pathophysiology of cystic fibrosis. Pediatr. Pulmonol. 50(Suppl 40): 2S4-S30 (2015).

20. Pezzulo, A. A. et al. Reduced airway surface $\mathrm{pH}$ impairs bacterial killing in the porcine cystic fibrosis lung. Nature 487, 109-113 (2012).

21. Quinton, P. M. Cystic fibrosis: impaired bicarbonate secretion and mucoviscidosis. Lancet 372, 415-417 (2008).

22. Pier, G. B. The challenges and promises of new therapies for cystic fibrosis. J. Exp. Med. 209, 1235-1239 (2012).

23. Meletiadis, J., Pournaras, S., Roilides, E. \& Walsh, T. J. Defining fractional inhibitory concentration index cutoffs for additive interactions based on self-drug additive combinations, Monte Carlo simulation analysis, and in vitro-in vivo correlation data for antifungal drug combinations against Aspergillus fumigatus. Antimicrob. Agents Chemother. 54, 602-609 (2010).

24. Tam, V. H., Schilling, A. N., Lewis, R. E., Melnick, D. A. \& Boucher, A. N. Novel approach to characterization of combined pharmacodynamic effects of antimicrobial agents. Antimicrob. Agents Chemother. 48, 4315-4321 (2004).

25. Meletiadis, J., Stergiopoulou, T., O'Shaughnessy, E. M., Peter, J. \& Walsh, T. J. Concentration-dependent synergy and antagonism within a triple antifungal drug combination against Aspergillus species: analysis by a new response surface model. Antimicrob. Agents Chemother. 51, 2053-2064 (2007).

26. Hoiby, N., Bjarnsholt, T., Givskov, M., Molin, S. \& Ciofu, O. Antibiotic resistance of bacterial biofilms. Int. J. Antimicrob. Agents 35, 322-332 (2010).

27. Stewart, P. S. Mechanisms of antibiotic resistance in bacterial biofilms. Int. J. Med. Microbiol. 292, 107-113 (2002).

28. Carter, W. H. Jr. Relating isobolograms to response surfaces. Toxicology 105 181-188 (1995).

29. Labiris, N. \& Dolovich, M. Pulmonary drug delivery. Part I: Physiological factors affecting therapeutic effectiveness of aerosolized medications. Br. J. Clin. Pharmacol. 56, 588-599 (2003).

30. Chase Watters, T. T. Y. \& Rumbaugh, K. P. Beneficial and deleterious bacterial-host interactions in chronic wound pathophysiology. Chron. Wound Care Manage. Res. 2, 53-62 (2015).

31. He, B. J. et al. Abstracts of Presentations at the International Conference on Basic and Clinical Multimodal Imaging $(\mathrm{BaCl})$, a Joint Conference of the International Society for Neuroimaging in Psychiatry (ISNIP), the International Society for Functional Source Imaging (ISFSI), the International Society for Bioelectromagnetism (ISBEM), the International Society for Brain Electromagnetic Topography (ISBET), and the EEG and Clinical Neuroscience Society (ECNS), in Geneva, Switzerland, September 5-8, 2013. Clin. EEG Neurosci., E1-E121 (2013).

32. Huse, H. K. et al. Parallel evolution in Pseudomonas aeruginosa over 39,000 generations in vivo. mBio 1, doi:10.1128/mBio.00199-10 (2010).

33. Aleksandrov, A. \& Field, M. Mechanism of activation of elongation factor Tu by ribosome: catalytic histidine activates GTP by protonation. RNA 19, 1218-1225 (2013).

34. Borovinskaya, M. A. et al. Structural basis for aminoglycoside inhibition of bacterial ribosome recycling. Nat. Struct. Mol. Biol. 14, 727-732 (2007).

35. Lazar, V. et al. Bacterial evolution of antibiotic hypersensitivity. Mol. Syst. Biol. 9, 700 (2013).

36. Taber, H. W., Mueller, J. P., Miller, P. F. \& Arrow, A. S. Bacterial uptake of aminoglycoside antibiotics. Microbiol. Rev. 51, 439-457 (1987).

37. Recinos, D. A. et al. Redundant phenazine operons in Pseudomonas aeruginosa exhibit environment-dependent expression and differential roles in pathogenicity. Proc. Natl Acad. Sci. USA 109, 19420-19425 (2012).

38. Colvin, K. M. et al. The pel polysaccharide can serve a structural and protective role in the biofilm matrix of Pseudomonas aeruginosa. PLoS Pathog. 7, e1001264 (2011).

39. Khan, W. et al. Aminoglycoside resistance of Pseudomonas aeruginosa biofilms modulated by extracellular polysaccharide. Int. Microbiol. 13, 207-212 (2010).

40. Hentzer, M. et al. Alginate overproduction affects Pseudomonas aeruginosa biofilm structure and function. J. Bacteriol. 183, 5395-5401 (2001).

41. Sezonov, G., Joseleau-Petit, D. \& D'Ari, R. Escherichia coli physiology in LuriaBertani broth. J. Bacteriol. 189, 8746-8749 (2007). 
42. Orhan, G., Bayram, A., Zer, Y. \& Balci, I. Synergy tests by E test and checkerboard methods of antimicrobial combinations against Brucella melitensis. J. Clin. Microbiol. 43, 140-143 (2005).

43. Sopirala, M. M. et al. Synergy testing by Etest, microdilution checkerboard, and time-kill methods for pan-drug-resistant Acinetobacter baumannii. Antimicrob. Agents Chemother. 54, 4678-4683 (2010).

44. Coffey, B. M. \& Anderson, G. G. Biofilm formation in the 96-well microtiter plate. Methods Mol. Biol. 1149, 631-641 (2014).

45. O'Toole, G. A. Microtiter dish biofilm formation assay. J Vis Exp e2437, doi:10.3791/2437 (2011).

46. Sabaeifard, P., Abdi-Ali, A., Soudi, M. R. \& Dinarvand, R. Optimization of tetrazolium salt assay for Pseudomonas aeruginosa biofilm using microtiter plate method. J Microbiol Methods 105, 134-140 (2014).
47. Joern, F. et al. Outcome for stage II and III rectal and colon cancer equally good after treatment improvement over three decades. Int J Colorectal Dis 30, 797-806 (2015).

This work is licensed under a Creative Commons Attribution 4.0 International License. The images or other third party material in this article are included in the article's Creative Commons license, unless indicated otherwise in the credit line; if the material is not included under the Creative Commons license, users will need to obtain permission from the license holder to reproduce the material. To view a copy of this license, visit http://creativecommons.org/licenses/ by/4.0/

Supplementary Information accompanies the paper on the npj Biofilms and Microbiomes website (http://www.nature.com/npjbiofilms) 